\title{
Novel ion conductive matrix via dehydrocoupling polymerization of imidazolium-type ionic liquid and lithium 9-borabicyclo[3,3,1]nonane hydride
}

\author{
Asako Narita, Wataru Shibayama, Noriyoshi Matsumi, and Hiroyuki Ohno(凶) \\ Department of Biotechnology, Tokyo University of Agriculture and Technology \\ 2-24-16 Naka-cho, Koganei, Tokyo, 184-8588, Japan
}

Received: 15 November 2005 / Revised version: 30 January 2006 / Accepted: 1 February 2006 Published online: 14 February 2006 - (C) Springer-Verlag 2006

\section{Summary}

An ion conductive zwitterionic polymer electrolyte containing borate units was prepared. The polymer was synthesized via dehydrocoupling of lithium 9borabicyclo[3,3,1]nonane hydride (lithium 9-BBN hydride) with 1,3dihydroxyethylimidazolium bromide and subsequent anion exchange reaction using lithium bis(trifluoromethylsulfonyl)imide (LiTFSI). The obtained polymer showed the ionic conductivity of $3.4 \times 10^{-6} \mathrm{Scm}^{-1}$ at $50{ }^{\circ} \mathrm{C}$ in spite of relatively high glass transition temperature $\left(-6^{\circ} \mathrm{C}\right)$.

\section{Introduction}

Ionic liquids (ILs) are interesting materials and potential candidates as ion conductive matrices. ILs show high ionic conductivity and negligible vapor pressure up to the decomposition temperature. Therefore, they are expected as potential electrolytes for electrochemical devices [1], in which lithium-cations [2], protons [3], or iodides (electrons) [4] should efficiently be transported.

However, in ILs containing salt or acid as carrier ion source, all the component ions migrate under potential gradient. High mobility of ions generally leads to low transference number of the added carrier ions. To overcome this problem, zwitterions in which anion and cation are covalently tethered were proposed [5]. For example, 1-(1ethyl-3-imidazolio)propane-3-sulfonate (EIm3S, Figure 1 a) melts at $176^{\circ} \mathrm{C}$. Although EIm3S was solid at room temperature, a mixture of EIm3S with equimolar amount of solid lithium bis(trifluoromethylsulfonyl)imide

(LiTFSI) turned liquid. This phenomenon is comprehended as the formation of IL-like environment between imidazolium cation and TFSI anion. The mixture of EIm3S and LiTFSI showed the ionic conductivity of $10^{-5} \mathrm{Scm}^{-1}$ at $50^{\circ} \mathrm{C}$ although pure zwitterion was an insulator [5].

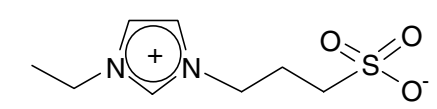

a. $\operatorname{EIm} 3 \mathrm{~S}$

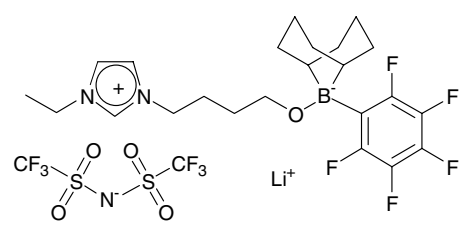

b. BZ/LiTFSI

Figure 1 Salts used 
Very recently, we have synthesized a low molecular weight borate-type zwitterion (BZ/LiTFSI, Figure 1 b) [6], which was prepared by a reaction of organoboron molten salt with organolithium reagent [7]. The obtained BZ/LiTFSI mixture showed the ionic conductivity of $3.0 \times 10^{-5} \mathrm{Scm}^{-1}$ at $50{ }^{\circ} \mathrm{C}$. Moreover, the transference number of lithium cation was 0.69 at room temperature, which is markedly high among IL systems without anion trapping unit.
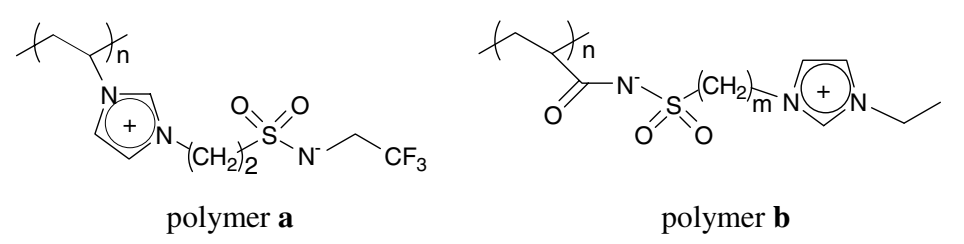

Figure 2 Pendant type polymerized zwitterions

Polymerization of zwitterions is a valuable approach to provide solid state ion conductive matrix [5]. However the pure polymerized zwitterions is insulator and no effective ion conduction is observed, mixtures of the polymerized zwitterions (Figure 2 polymer b) with equimolar amount of LiTFSI to polymer unit showed ionic conductivity of around $10^{-5} \mathrm{Scm}^{-1}$ at $50^{\circ} \mathrm{C}$.

In the present work, we have prepared zwitterionic polymer bearing lithium borate units as anionic sites. Here, preparation of polymerized zwitterion was examined by dehydrocoupling polymerization developed by Chujo et al [8]. We have recently extended this method to a synthesis of polyether based organoboron polymer electrolytes [9]. In this study, dehydrocoupling polymerization between 1,3dihydroxyethylimidazolium bromide and lithium 9-BBN hydride and subsequent anion exchange reaction with LiTFSI afforded the corresponding polymer composed of zwitterionic structure.

\section{Experimental section}

\section{Materials}

The polymer was prepared as seen in Figure 3. 1,3-Dihydroxyethyl- imidazolium bromide in Figure 3 was prepared by the reported procedures [10,11]. Lithium 9BBN hydride tetrahydrofuran $1.0 \mathrm{M}$ solution (Aldrich, $2.4 \mathrm{ml}$ ) was slowly added to $0.54 \mathrm{~g}$ of 1,3-dihydroxyethylimidazolium bromide under argon gas atmosphere at $0{ }^{\circ} \mathrm{C}$. The mixture was stirred for 24 hours at room temperature. After tetrahydrofuran was removed under reduced pressure, the crude polymer was reprecipitated from methanol in n-hexane. The obtained polymer was dried under reduced pressure for 3 days at room temperature. The polymer $(0.76 \mathrm{~g})$ was mixed with $0.60 \mathrm{~g}$ LiTFSI $(99.97 \%$ from Sumitomo $3 \mathrm{M}$ ) in $10 \mathrm{ml}$ tetrahydrofuran, and the mixture was stirred for 2 days. After tetrahydrofuran was removed under reduced pressure, $30 \mathrm{ml}$ of the mixture of acetonitrile and dichloromethane (3:1 by volume) was added. After the solution was further stirred for 3 days, soluble fraction was collected by syringe. After solvent was removed by evaporation, the resulting polymer was dried for 3 days to give the polymer c. ${ }^{1} \mathrm{H}-\mathrm{NMR}$ : $\delta_{\mathrm{H}}\left(\mathrm{CD}_{3} \mathrm{OD}\right.$, ppm, relative to $\left.\mathrm{Me}_{4} \mathrm{Si}\right) 1.24(2 \mathrm{H}$ x 2, 9-BBN), $1.56\left(2 \mathrm{H} \mathrm{x} \mathrm{4,} \mathrm{9-BBN),} 1.80\left(2 \mathrm{H} \mathrm{x} \mathrm{2,9-BBN),} 3.85\left(2 \mathrm{H} \mathrm{x} 2, \mathrm{~N}-\mathrm{CH}_{2} \mathrm{CH}_{3} \mathrm{O}\right), 4.29(2 \mathrm{H} \mathrm{x}\right.\right.$ $\left.2, \mathrm{~N}-\mathrm{CH}_{2} \mathrm{CH}_{3} \mathrm{O}\right)$, and $7.62(1 \mathrm{H} \mathrm{x} 2$, imidazolium $)$. 


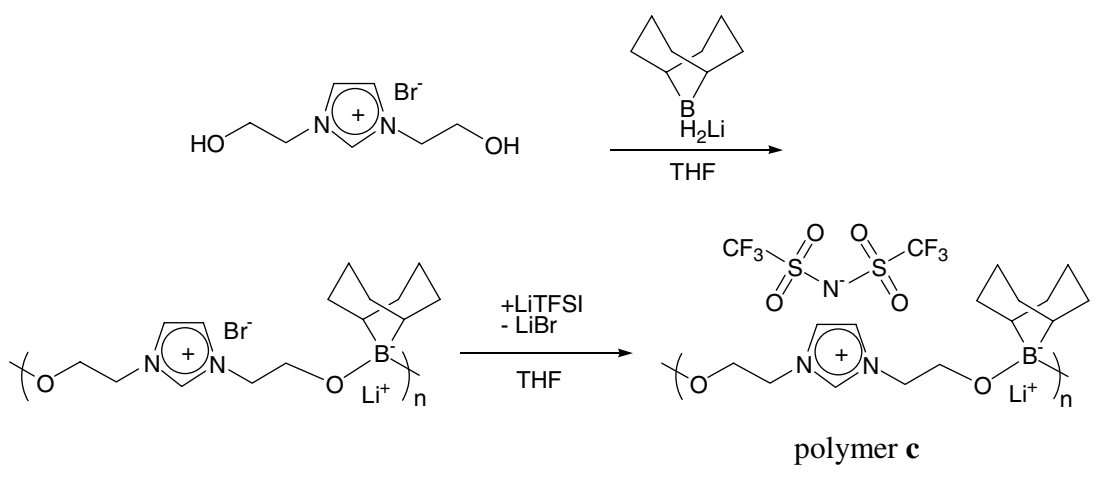

Figure 3 Polymerized zwitterion containing LiTFSI

\section{Measurements}

The glass transition temperature $\left(T_{\mathrm{g}}\right)$ was measured on differential scanning calorimetry (DSC, SII DSC EXSTAR 6000, Seiko Instruments) over a range from $-120{ }^{\circ} \mathrm{C}$ to $200{ }^{\circ} \mathrm{C}$. Ionic conductivity was measured by complex impedance method on Impedance/Gain-phase analyzer (Solartron 1260, Schlumberger) under dried nitrogen gas atmosphere over a range from $10^{\circ} \mathrm{C}$ to $60^{\circ} \mathrm{C}$.

\section{Results and Discussion}

The zwitterionic organoboron polymer containing LiTFSI, polymer $\mathbf{c}$ in Figure 3, was obtained as a pale yellow powder that was soluble in common organic solvents such as tetrahydrofuran, methanol, and so on. The excellent solubility of this polymer implies that closs-linking of the polymer via intramolecular ion exchange did not take place. After cast from methanol solution, the appearance of the polymer c was slightly colored transparent amorphous solid.

From the ${ }^{11} \mathrm{~B}-\mathrm{NMR}$ spectrum, one main peak due to dialkoxyborate was observed at $-15.5 \mathrm{ppm}\left(\mathrm{CD}_{3} \mathrm{OD}\right.$, relative to $\mathrm{BF}_{3} \mathrm{OEt}_{2}$, Figure 4), in the typical chemical shift region for borate structure. The shape of the peak was sharp and monomodal. This also indicates that anion exchange reaction between polymer units did not take place. From the ${ }^{1} \mathrm{H}-\mathrm{NMR}$ spectrum, no peak due to hydroxyl group was observed, while peaks owing to $9-\mathrm{BBN}$ structure were observed in $1.24 \sim 1.80 \mathrm{ppm}$. The integration ratio for all the peaks agreed well with those for expected structure.

Since the peak attributable to terminal methoxyborate was observed around 3.6 ppm in the ${ }^{1} \mathrm{H}-\mathrm{NMR}$ spectrum, it was indicated that cyclization did not prevail in the present polymerization system. From the integration of the peak due to terminal methoxyborate unit around $3.6 \mathrm{ppm}$ and the peak owing to methylene group adjacent to imidazolium ring, the number of repeating units in polymer $\mathbf{c}$ was calculated to be 5.2 , which was similar to those for other organoboron polymer electrolytes prepared by dehydrocoupling polymerization.

From the DSC measurement, no decomposition of polymer $\mathbf{c}$ was observed below $200{ }^{\circ} \mathrm{C}$. Such thermal stability of polymer $\mathbf{c}$ might be due to severe steric hindrance around the boron atom. The polymer $\mathbf{c}$ showed $T_{\mathrm{g}}$ at $-6^{\circ} \mathrm{C}$, which was higher than those for polymers bearing zwitterions in their side chain $\left(-16 \sim-33{ }^{\circ} \mathrm{C}\right.$, polymers $\mathbf{a}$ 
and $\mathbf{b}$ in Figure 2 [5]). The $T_{\mathrm{g}}$ of polymer $\mathbf{c}$ was also higher than those for other polymerized organoboron ionic liquids, possibly because of some intramolecular electrostatic interactions. No peak other than $T_{\mathrm{g}}$ was observed during the DSC measurement, indicating amorphous nature of polymer $\mathbf{c}$.

Ionic conductivity of polymer $\mathrm{c}$ was evaluated by ac impedance method after sandwiched between stainless steel electrodes. Before the measurement of ionic conductivity, the sample was thoroughly dried under vacuum. Similar to the case of polymer electrolytes bearing a mesitylboron group, the presence of bulky structure in the main chain did not prevent efficient ionic conduction. As seen in the Arrhenius plot represented in Figure 5, the ionic conductivity of polymer c monotonously increased with elevating temperature. This also demonstrates the reasonable stability of polymer $\mathrm{c}$ in the observed temperature range.

The ionic conductivity of polymer $\mathbf{c}$ was found to be $3.4 \times 10^{-6} \mathrm{Scm}^{-1}$ at $50{ }^{\circ} \mathrm{C}$. Generally, ionic conductivity of polymer electrolytes is inversely proportional to $\mathrm{Tg}$ of polymers. However, it is interesting to note that moderate ionic conductivity was observed in the present system in spite of relatively high $T_{\mathrm{g}}\left(-6^{\circ} \mathrm{C}\right)$ of the polymer. Furthermore, the Arrhenius plots for polymer c showed small temperature dependence of ionic conductivity. The observed decoupling of ionic conductivity with thermal behavior indicates that ionic conduction in polymer c does not depend only on segmental motion of the polymer. The presence of excellent ionic liquid structure (imidazolium-TFSI) is considered to provide polar microenvironment, which might be responsible for moderate ionic conductivity in spite of high $T_{\mathrm{g}}$.

It is generally of much interest to design ion conductive matrices showing both high ionic conductivity and good mechanical property at the same time. Our results demonstrate that synthesis of relatively rigid zwitterionic polymer including a good ionic liquid structure and dissociable lithium salt structure can be a valuable approach for such materials.

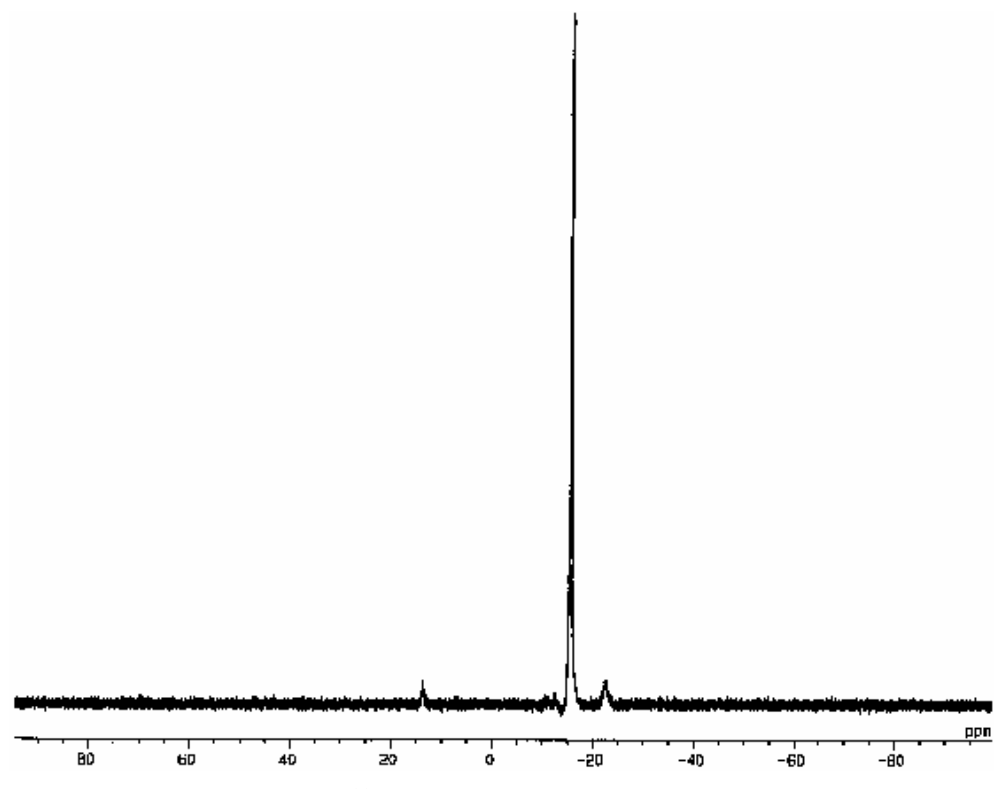

Figure $4{ }^{11} \mathrm{~B}-\mathrm{NMR}$ spectrum for polymer $\mathrm{c}$ 


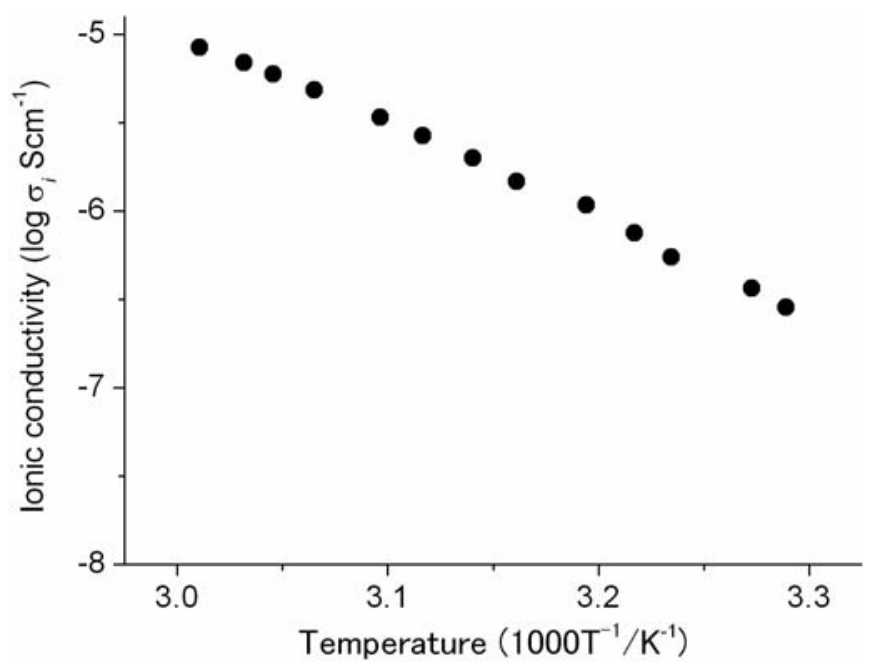

Figure 5 The temperature dependence of ionic conductivity for zwitterionic polymer c

In conclusion, novel zwitterionic polymer electrolyte bearing lithium borate structure was prepared. A dehydrocoupling polymerization of an ionic liquid monomer using commercially available lithium 9-BBN hydride resulted in successful formation of the corresponding polymer composed of 5.2 repeating units. The preparation was accompanied by neither cyclization nor intramolecular ion exchange to afford the desired polymer that was well soluble in common organic solvents. Further combination of ionic liquid and boron hydride would produce a variety of zwitterionic polymer bearing lithium borate structure. The interesting feature of the obtained polymer electrolyte was ionic conductivity over $10^{-6} \mathrm{~S} / \mathrm{cm}$ at $50^{\circ} \mathrm{C}$ despite of high $T_{\mathrm{g}}\left(-6^{\circ} \mathrm{C}\right)$. The observed decoupling of ionic conductivity with thermal behavior shows ion conductive mechanism is not closely dependent on segmental motion of matrices. This phenomenon implies that arrangement of good ionic liquid structure and dissociable lithium borate on relatively rigid polymer backbone was a fruitful approach to design polymers showing both high ionic conductivity and good mechanical property. Synthesis of zwitterionic polymer bearing more highly dissociable lithium borate structure is also underway.

Acknowledgements. The financial support for this study by a Grant-in-Aid for Scientific Research from the Ministry of Education, Culture, Sports, Science and Technology, Japan (No. 17205020 and 17073005) is gratefully acknowledged. LiTFSI was a gift from Sumitomo 3M. The present study was carried out under the 21st COE program of Future Nano-Materials.

\section{References}

1. Ed: Ohno H (2005) Electrochemical Aspects of Ionic Liquids: Wiley-Interscience

2. For example, Carlin T, De Long. C, Fuller, J, Trulove C (1994) J Electrochem Soc 141: L73

3. For example, Doyle M, Choi S-K, Proulx G (2000) J Electrochem. Soc 147: 34

4. For example, Bonhôte P, Dias A-P, Papageorgiou N, Kalyanasundaram K and Grätzel M (1996) Inorg Chem 36: 1168 
5. Yoshizawa M, Hirao M, Ito-Akita K and Ohno H (2001) J Mater Chem 11: 1057

6. Narita A, Shibayama W, Ohno H (2006) in contribution

7. Matsumi N, Miyake M, Ohno H (2003) Chem Commn: 2852

8. Chujo Y, Tomita I, Suegusa T (1991) Polym J 23: 743

9. a. Matsumi N, Sugai K, Ohno H (2003) Macromolecules 36: 2321

b. Matsumi N, Sugai K, Sakamoto K, Mizumo T, Ohno H (2005) Macromolecules 38: 4951

10. Yoshino T, Inaba S, Komura H, Ishida Y (1977) J Chem Soc Perkin Trans 1 11: 1266

11. Herrmann W A, Goosen L J, Spiegler M (1997) J Organomet Chem 547: 357 